\title{
A note on the generalized fractal dimensions of a probability measure
}

\author{
Charles-Antoine Guérin ${ }^{\text {a) }}$ \\ Institut Fresnel, UMR CNRS 6133, Faculté des Sciences de Saint-Jérôme, \\ Case 162, F-13397 Marseille Cedex 20, France
}

\begin{abstract}
We prove the following result on the generalized fractal dimensions $D_{q}^{ \pm}$of a probability measure $\mu$ on $\mathbb{R}^{n}$. Let $g$ be a complex-valued measurable function on $\mathbb{R}^{n}$ satisfying the following conditions: (1) $g$ is rapidly decreasing at infinity, (2) $g$ is continuous and nonvanishing at (at least) one point, (3) $\int g \neq 0$. Define the partition function $\Lambda_{a}(\mu, q)=a^{n(q-1)}\left\|g_{a} * \mu\right\|_{q}^{q}$, where $g_{a}(x)=a^{-n} g\left(a^{-1} x\right)$ and $*$ is the convolution in $\mathbb{R}^{n}$. Then for all $q>1$ we have $D_{q}{ }^{ \pm}=1 /(q-1) \lim _{r \rightarrow 0} \inf ^{\text {sup }} \times\left[\log \Lambda_{a} \mu(r, q) /\right.$ $\log r]$.
\end{abstract}

\section{INTRODUCTION}

Since the apparition of the fractal formalism in the late 1970s, there has been a huge amount of literature devoted to the different definitions of the fractal dimensions of probability measures (see, e.g., Refs. 1 and 2 for reviews). Roughly speaking, there are two types of fractal dimensions: the pointwise dimensions, which give essentially the local Hölder exponents of the measure, and the global ones, which can be seen as regularity indices in a scale of Besov spaces. Both families of dimensions are related to one another by the so-called multifractal formalism. The global dimensions were originally introduced by Renyi ${ }^{3}$ and rediscovered by Hentschel and Procaccia in their seminal paper; ${ }^{4}$ they are a generalization of the usual fractal dimension or capacity, and are therefore called "generalized fractal dimensions." They are obtained by a box-counting algorithm, which amounts to partitioning the space in elementary cells and suming up powers of the individual contributions to the measure into what is called a partition function. There are several variants of this definition, according to the kind of covering that is chosen (grids, balls, redundant, nonredundant, etc.) and the kind of partition function (discrete or continuous). First used in the context of chaos and dynamical systems, the generalized fractal dimensions have regained interest in the framework of quantum diffusion in the presence of fractal spectra. After the pioonering work of Guarneri ${ }^{5}$ many relations were established between the diffusive behavior of quantum wave packets and the generalized fractal dimensions (see, e.g., Refs. 6-9 for some recent results).

In order to simplify the numerous definitions and unify the results arising in dimension theory, it is important to find equivalences between the different approaches. This has been the aim of some recent works (e.g., Refs. 10-12, 2). In this short note we show that the generalized fractal dimensions can be obtained by replacing the boxes by arbitrary rapidly decaying complex-valued functions in the partition function, provided only these functions have nonzero mean.

\section{THE GENERALIZED FRACTAL DIMENSIONS}

Let $\mu$ be a probability Borel measure on $\mathbb{R}^{n}$ and consider a partition of the space in a grid of cubes $\mathcal{Q}\left(x_{j}, r\right)$ of center $x_{j}$ and side $2 r$. For $q \geqslant 0$, form the following partition function:

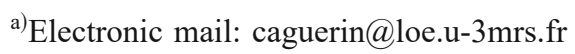




$$
\Lambda_{1} \mu(r, q)=\sum_{j} \mu\left(\mathcal{Q}\left(x_{j}, r\right)\right)^{q}
$$

The so-called Rényi dimensions $D_{q}^{ \pm}$(Ref. 4) of the measure $\mu$ are defined as the limiting exponents of the last function as $r \rightarrow 0$, precisely:

$$
D_{q}^{ \pm}=\frac{\tau_{q}^{ \pm}}{q-1}, \quad q \neq 1
$$

with

$$
\tau_{q}^{ \pm}=\lim _{r \rightarrow 0} \sup _{\inf } \frac{\log \Lambda_{1} \mu(r, q)}{\log r}
$$

This normalization recasts the dimensions on the unit interval, $0 \leqslant D_{q}^{ \pm} \leqslant 1$, at least for $q>1$ (see Ref. 12 for a detailed discussion). Note that (2.2) is not defined for $q=1$. Hentschel and Proccacia ${ }^{4}$ have shown heuristically the existence of the limit $q \rightarrow 1$, thereby defining $D_{1}^{ \pm}$by continuity. A rigorous analysis ${ }^{12}$ however, shows that $D_{q}^{ \pm}$are only left- and right-continuous about $q=1$, with a possible discontinuity. The dimension $D_{0}^{ \pm}$is the usual fractal dimension or capacity. The dimension $D_{2}^{ \pm}$is known as the correlation dimension. A continuous version ${ }^{4,10}$ of the partition function (2.1) is

$$
\Lambda_{2} \mu(r, q)=\int_{\mathbb{R}^{n}} \mu(\mathcal{B}(x, r))^{q-1} \mathrm{~d} \mu(x)
$$

where $\mathcal{B}(x, r)$ is the ball of center $x$ and radius $r$. The corresponding limiting exponents, defined after (2.2) and (2.3), are called generalized fractal dimensions. Note that the integration is performed against the measure $\mu$, which is possibly singular. A more tractable definition consists in integrating versus the Lebesgue measure, by formally replacing $\mathrm{d} \mu(x)$ by the absolutely continuous measure $r^{-n} \mu(\mathcal{B}(x, r))$ :

$$
\Lambda_{3} \mu(r, q)=r^{-n} \int_{\mathbb{R}^{n}} \mu(\mathcal{B}(x, r))^{q} \mathrm{~d} x .
$$

Although more convenient, this formula seems to be less popular in the literature. The equivalence of definitions (2.1) and (2.4) has been shown in Ref. 13 for $q>1$ (also rewritten in Ref. 2, p. 184), and the equivalence of (2.1) and (2.5) follows by an obvious adaptation of the proof. The equivalence for $0<q<1$ has been proved more recently. ${ }^{12}$ We thus have, for all $q>0(q \neq 1)$, three equivalent definitions of the generalized fractal dimensions:

$$
D_{q}^{ \pm}=\frac{1}{q-1} \lim _{r \rightarrow 0} \sup _{\inf } \frac{\log \Lambda_{j \mu}(r, q)}{\log r}, \quad j=1,2,3 .
$$

\section{MAIN RESULT}

As we are going to show, the balls or boxes used to define the partition functions $\Lambda_{j} \mu$ can be replaced by arbitrary measurable functions, provided the latter are rapidly decreasing. This is very natural for non-negative functions, which can be seen as smooth cutoff functions, but less obvious for complex or signed functions. For any measurable complex-valued function $g$ on $\mathbb{R}^{n}$ denote by $g_{a}(x)=a^{-n} g\left(a^{-1} x\right), a>0$, its dilated version and define the partition function

$$
\Lambda_{a}(\mu, q)=a^{n(q-1)}\left\|g_{a} * \mu\right\|_{q}^{q}=a^{n(q-1)} \int \mathrm{d} b\left|g_{a} * \mu(b)\right|^{q},
$$


where the asterisk $(*)$ stands for the convolution:

$$
g_{a} * \mu(b)=\int g_{a}(b-x) \mathrm{d} \mu(x),
$$

We start by recalling a useful rule of computation for the limiting exponents. A proof of this result can be found in Ref. 14, for example.

Lemma 3.1: Let s be a non-negative measurable function. Then

$$
\begin{gathered}
\liminf _{t \rightarrow 0} \frac{\log s(t)}{\log t}=\sup \left\{\gamma: s t(t) \leqslant O\left(t^{\gamma}\right), t \rightarrow 0\right\}, \\
\limsup _{t \rightarrow 0} \frac{\log s(t)}{\log t}=\inf \left\{\gamma: t^{\gamma} \leqslant O(s(t)), t \rightarrow 0\right\} .
\end{gathered}
$$

Next we consider the case of non-negative functions.

Lemma 3.2: Let $g$ be a non-negative measurable function $g$ on $\mathbb{R}^{n}$ which is continuous and nonvanishing at (at least) one point and rapidly decreasing at infinity. Then for all $q>1$ we have

$$
D_{q}^{ \pm}=\frac{1}{q-1} \lim _{r \rightarrow 0} \sup _{\inf } \frac{\log \Lambda_{a} \mu(r, q)}{\log r} .
$$

Proof: First note that we do not change the limit (3.1) on replacing $g$ by a translated and rescaled version $\lambda g(\alpha t-\beta)$. Since $g$ remains positive in some neighborhood of, say, $t_{0}$, we can rescale $g$ in such a way that $c h \leqslant g \leqslant C h$, where $h$ is some non-negative function with $h(t)=1$ for $t \in[-1,1]$ and $c, C$ are two positive constants. Hence it suffices to prove the lemma for such a function $h$. Let $\epsilon>0$. For all $a>0$ small enough and $b \in \mathbb{R}$ we have

$$
\begin{aligned}
\left|h_{a} * \mu(b)\right| & =\int h_{a}(t) \mathrm{d} \mu(b-t) \\
& \geqslant \int_{|t| \leqslant a^{1+\epsilon}} h_{a}(t) \mathrm{d} \mu(b-t) \\
& \geqslant a^{-n} \min _{|t| \leqslant a} \epsilon\{h(t)\} \mu\left(\mathcal{B}\left(b, a^{1+\epsilon}\right)\right) \\
& \geqslant a^{-n} \mu\left(\mathcal{B}\left(b, a^{1+\epsilon}\right)\right) .
\end{aligned}
$$

Integrating over $b$ this yields to

$$
\left\|h_{a} * \mu\right\|_{q}^{q} \geqslant a^{(1-q) n+\epsilon n} \Lambda_{3} \mu\left(a^{1+\epsilon}, q\right) .
$$

The reverse estimation is more touchy. For all $\epsilon>0$ we have

$$
\begin{aligned}
\left|h_{a} * \mu(b)\right| & \leqslant \int_{|t| \leqslant a^{1-\epsilon}}\left|h_{a}(t)\right| \mathrm{d} \mu(b-t)+\int_{|t|>a^{1-\epsilon}}\left|h_{a}(t)\right| \mathrm{d} \mu(b-t) \\
& \leqslant a^{-n}\|g\|_{\infty} \mu\left(\mathcal{B}\left(b, a^{1-\epsilon}\right)\right)+\int_{|t|>a^{1-\epsilon}}\left|h_{a}(t)\right| \mathrm{d} \mu(b-t)
\end{aligned}
$$

and thus

$$
\left\|h_{a} * \mu\right\|_{q}^{q} \leqslant C_{q} a^{(1-q) n-\epsilon n} \Lambda_{3} \mu\left(a^{1-\epsilon}, q\right)+C_{q} \int \mathrm{d} b\left(\int_{|b-t|>a^{1-\epsilon}} h_{a}(b-t) \mathrm{d} \mu(t)\right)^{q}
$$


for some constant $C_{q}$. We have to show that the contribution of the second term is negligible. By Jensen's inequality, we have for all $q>1$,

$$
\left(\int_{|t|>a^{1-\epsilon}} h_{a}(t) \mathrm{d} \mu(b-t)\right)^{q} \leqslant\left(\int_{|b-t|>a^{1-\epsilon}} \mathrm{d} \mu(t)\right)^{q-1} \int_{|b-t|>a^{1-\epsilon}} h_{a}^{q}(b-t) \mathrm{d} \mu(t) .
$$

Thus, calling $I(a)$ the second term on the right-hand side of (3.3), we have

$$
\begin{aligned}
I(a) & \leqslant C_{q} \int \mathrm{d} b \int_{|t|>a^{1-\epsilon}} h_{a}^{q}(t) \mathrm{d} \mu(b-t) \\
& =C_{q} \int_{|t|>a^{1-\epsilon}} h_{a}^{q}(t) \int \mathrm{d} \mu(b-t)=C_{q} a^{(1-q) n} \int_{|t|>a^{-} \epsilon} g^{q}(t),
\end{aligned}
$$

where we use the finiteness of $\mu$ to exchange the integrals. Now since $g$ is rapidly decreasing at infinity, the tails of its integrals also and thus $I(a) \leqslant O\left(a^{\infty}\right), a \rightarrow 0$. Since (3.2) and (3.3) hold for arbitrarily small $\epsilon$, the conclusion follows from Lemma 3.1.

Note: A proof of this result in the case $0<q<1$ has been given in Ref. 12, Theorem A.1.

We now can state the main result:

Theorem 3.3: Let $g$ be a complex-valued measurable function on $\mathbb{R}^{n}$ satisfying the following conditions:

(1) $g$ is rapidly decreasing at infinity,

(2) $g$ is continuous and nonvanishing at (at least) one point,

(3) $\int g \neq 0$.

Then for all $q>1$ we have

$$
D_{q}^{ \pm}=\frac{1}{q-1} \lim _{r \rightarrow 0} \sup _{\inf } \frac{\log \Lambda_{a} \mu(r, q)}{\log r} .
$$

This will be an easy consequence of the following lemma.

Lemma 3.4: Let $g$ be a rapidly decreasing complex-valued measurable function such that $\int g \neq 0$. Then there exists a non-negative function $\phi$ in $\mathcal{S}\left(\mathbb{R}^{n}\right)$ (the Schwartz space of infinitely differentiable rapidly decreasing functions) and a complex-valued function $\psi \in \mathcal{S}\left(\mathbb{R}^{n}\right)$ such that $\phi=g^{*} \psi$.

Proof: Let $\hat{g}$ be the notation for the Fourier transform of a $L^{1}$ function $g$ :

$$
\hat{g}(k)=\int e^{-i k x} g(x) \mathrm{d} x
$$

Without restriction we may suppose $\left|\int g\right|=|\hat{g}(0)|=1$. Since $\hat{g}$ is a continuous function, we can find some neighborhood $V=B(0, \epsilon)$ about the origin in which $|\hat{g}|>1 / 2$ on $V$. Now take a smaller neighborhood $V^{\prime}=B(0, \epsilon / 2)$ and some nonzero $h \in C_{0}^{\infty}\left(\mathbb{R}^{n}\right)$ with support in $V^{\prime}$. Define $\phi$ by its Fourier transform: $\hat{\phi}=h * \widetilde{h}$, where $\widetilde{h}(x)=\bar{h}(-x)$ and $\bar{h}$ is the complex conjugate of $h$. Then support $(\hat{\phi}) \subset V$ and $\phi=|\hat{h}|^{2}$ is a non-negative function in $\mathcal{S}\left(\mathbb{R}^{n}\right)$. It remains to construct $\psi$. This can be done by setting $\hat{\psi}=\hat{\phi} / \hat{g}$ on $V, \hat{\psi}=0$ elsewhere. The two functions then satisfy $\phi=g * \psi$ and $\psi$ is in $\mathcal{S}\left(\mathbb{R}^{n}\right)$ for $\hat{\psi}$ is in $C_{0}^{\infty}\left(\mathbb{R}^{n}\right)$.

Proof (of Theorem 3.3): Take two functions $\phi$ and $\psi$ as in Lemma 3.4. Then for all $q>1$, we have by Young's inequality:

$$
\left\|\mu * \phi_{a}\right\|_{q}=\left\|\left(\mu * g_{a}\right) * \psi_{a}\right\|_{q} \leqslant\left\|\mu * g_{a}\right\|_{q}\|\psi\|_{1} .
$$

On the other hand, 


$$
\left\|\mu * g_{a}\right\|_{q} \leqslant\left\|\mu *\left|g_{a}\right|\right\|_{q} .
$$

Both functions $\phi$ and $|g|$ fulfill the hypothesis of Lemma 3.2. The partition functions $a^{n(q-1)}\left\|\mu * \phi_{a}\right\|_{q}$ and $a^{n(q-1)}\left\|\mu *\left|g_{a}\right|\right\|_{q}$ therefore satisfy (2.6), and since they "sandwich" $\Lambda_{a}(\mu, q)$, the conclusion follows.

\section{ACKNOWLEDGMENTS}

Most of this work has been done at the Center for Dynamical Systems, Como, under European Grant No. ERB4001GT974293. Many thanks go to G. Mantica and I. Guarneri for useful discussions, and to J.M. Barbaroux for useful comments on the manuscript.

${ }^{1}$ C. D. Cutler, "A review of the theory and estimation of fractal dimension," in Dimension Estimation and Models (World Scientific, River Edge, NJ, 1993) pp. 1-107.

${ }^{2}$ Ya. B. Pesin, Dimension Theory in Dynamical Systems, Chicago Lectures in Mathematics (The University of Chicago Press, Chicago, 1997).

${ }^{3}$ A. Rényi, Probability Theory (North-Holland, Amsterdam, 1970).

${ }^{4}$ H. G. E. Hentschel and I. Procaccia, "The infinite number of generalized dimensions of fractals and strange attractors," Physica D 8, 435-444 (1983).

${ }^{5}$ I. Guarneri, "Spectral properties of quantum diffusion on discrete lattices," Europhys. Lett. 10, 95-100 (1989).

${ }^{6}$ J. M. Barbaroux, J. M. Combes, and R. Montcho, "Remarks on the relation between quantum dynamics and fractal spectra,” J. Math. Anal. Appl. 213, 698-722 (1997).

${ }^{7}$ J. M. Barbaroux and H. Schulz-Baldes, “Anomalous quantum transport in presence of self-similar spectra,” Ann. I.H.P. Phys. Theor. 71, 1-21 (1999).

${ }^{8}$ G. Mantica, "Quantum intermittency in almost periodic systems derived from their spectral properties," Physica D 103, 576-589 (1997)

${ }^{9}$ I. Guarneri and H. Schulz-Baldes, "Intermittent lower-bounds on quantum diffusion," Lett. Math. Phys. 49, 317-324 (1999).

${ }^{10}$ Ya. B. Pesin, “On rigorous mathematical definition of the correlation dimension and generalized spectrum for dimension," J. Stat. Phys. 71, 529-547 (1993).

${ }^{11}$ C. A. Guérin and M. Holschneider, "On equivalent definitions of the correlation dimension for a probability measure,' J. Stat. Phys. 86, 707-720 (1997).

${ }^{12}$ J. M. Barbaroux, F. Germinet, and S. Tcheremchantsev, "Generalized fractal dimensions: Equivalences and basic properties," Technical Report No. 00-341, http://www.ma.utexas.edu/mparch.

${ }^{13}$ M. Guysinsky and S. Yaskolko, "Coincidence of various dimensions associated with metrics and measures on metric spaces,” Discrete Contin. Dyn. Systems 3, 591-603 (1997).

${ }^{14}$ M. Holschneider, "Fractal wavelet dimension and localization," Commun. Math. Phys. 160, 457-473 (1994). 